\title{
Clock position-based iris bow configuration after laser peripheral iridotomy in Chinese angle closure eyes: a swept source optical coherence tomography study
}

\author{
Shida Chen ${ }^{1} \cdot$ Yaoming $\mathrm{Liu}^{1} \cdot$ Fei $\mathrm{Li}^{1} \cdot$ Kai Gao ${ }^{1} \cdot$ Rouxi Zhou ${ }^{1} \cdot$ Xiulan Zhang $\mathbb{1}^{1}$
}

Received: 17 December 2018 / Revised: 25 June 2019 / Accepted: 11 September 2019 / Published online: 25 September 2019

(c) The Author(s), under exclusive licence to The Royal College of Ophthalmologists 2019

\begin{abstract}
Background To determine how many measurements should be evaluated to determine the iris bow and evaluate changes of iris bow at 12 clock positions after LPI in primary angle closure eyes.

Methods A total of 93 primary angle closure eyes in 93 Chinese patients were enrolled. Anterior iris bowing was evaluated at 12 clock positions and 4 clock positions (3, 6, 9, and 12 o'clock) before, 1 week and 3 months after LPI using swept source optical coherence tomography.

Results At baseline, almost all of the eyes exhibited an iris bow when measured using 12 clock positions, consistent with results obtained from measurements at 4 clock positions (Cronbach's alpha $=0.99)$. LPI caused a relative unified change in all of the clock positions (Cronbach's alpha $=0.91$ ) except the LPI site. After LPI, there was no significant difference between 12 and 4 clock position measurements for the iris bow (both $p>0.05$ ), with $34.1 \%$ vs. $33 \%$ of the patients remained iris bow at 1 week and $34 \%$ vs. $31.9 \%$ of the patients remained iris bow at 3 months. However, the coexisting iris bow configuration was more common when measured using 4 clock positions ( $16.5 \%$ vs. $3.3 \%$ at 1 week and $25.5 \%$ vs. $10.6 \%$ at 3 months).

Conclusions There was excellent consistency when measuring the iris bow at 4 or 12 clock positions. LPI caused a relatively unified iris bow change at 12 clock positions, and a single LPI relieved only $2 / 3$ of the iris bow configurations.
\end{abstract}

\section{Introduction}

More sophisticated and objective methods, including ultrasonographic biomicroscopy (UBM) and anterior segment optical coherence tomography (AS-OCT), have been widely used in the assessment of the ocular anterior segment in angle closure eyes $[1,2]$. AS-OCT provides objective highresolution visualization and reproducible measurements of the anterior segment structures and has been used to describe the novel anatomical features associated with angle closure,

These authors contributed equally: Shida Chen, Yaoming Liu

Supplementary information The online version of this article (https:// doi.org/10.1038/s41433-019-0601-1) contains supplementary material, which is available to authorized users.

Xiulan Zhang

zhangx12@mail.sysu.edu.cn

1 Zhongshan Ophthalmic Center, State Key Laboratory of Ophthalmology, Sun Yat-Sen University, Guangzhou, People's Republic of China such as a smaller anterior chamber width, area, volume, iris curvature, and lens vault $[3,4]$.

Laser peripheral iridotomy (LPI) is the recommended first-line treatment for angle closure eyes, including primary angle closure suspect (PACS), primary angle closure (PAC), and primary angle closure glaucoma (PACG) eyes, especially for relieving iris bow. It can also serve as an intervention to prevent the onset of acute angle closure and the development of optic nerve damage [5-7]. However, a single LPI is not sufficient to prevent angle closure progression in all eyes $[6,8]$. One of the reasons may be due to persistent iridotrabecular contact (ITC), which occurs in more than $20 \%$ of eyes after LPI [9]. Therefore, we need a technology to fully examine changes in the anterior segment in these eyes. Previously, AS-OCT or UBM have been shown to be able to obtain only the horizontal and vertical data of the anterior segment unless the subject moved their eyes or turned their head in different directions. Furthermore, most previous studies analyzed only horizontal data [10-13], leaving whole changes in the iris configuration unexplored. Advances have been made in OCT technology, and the new swept source OCT (SS-OCT) allows a 
$360^{\circ}$ scan of the anterior chamber, thereby providing more comprehensive information about anterior chamber angle structures.

The objective of this study was to use SS-OCT evaluate changes in iris morphology at 12 clock positions after LPI in patients with angle closure. In addition, whether evaluating the iris configuration based on horizontal and vertical data could replace the use of a 12 clock position configuration was also determined.

\section{Methods}

\section{Subject recruitment}

This prospective study consecutively recruited 91 patients diagnosed with primary angle closure diseases, including PACG, PAC, and PACS, between September 2016 and December 2017 from the glaucoma clinics of Zhongshan Ophthalmic Center of Sun Yat-sen University in Guangzhou, China. The eligible patients were scheduled for LPI. This study was approved by the Ethical Review Committee of the Zhongshan Ophthalmic Center, and it was conducted in accordance with the tenets of the Declaration of Helsinki. All of the participants received detailed explanations about the study, and a signed consent form was obtained from every participant.

Each participant underwent a comprehensive ophthalmic examination, including slit lamp biomicroscopy, applanation tonometry, gonioscopy, fundus examination, and visual field examination (24-2 test pattern, Humphrey Visual Field Analyzer II; Carl Zeiss Meditec, Inc., Dublin, CA, USA). The diagnosis of PACS was determined as follows: eyes with ITC of $180^{\circ}$ or more in the primary gaze upon gonioscopy that were without PAS, had a normal IOP, normal-appearing optic discs, and a normal visual field. The diagnosis of PAC was determined as follows: eyes with ITC of at least $180^{\circ}$ or more and an elevated IOP or PAS with no secondary cause for the PAS and without glaucomatous optic neuropathy. The diagnosis of PACG was determined as follows: narrow angles with glaucomatous optic neuropathy. Evidence of glaucomatous optic neuropathy was defined as a vertical cup-disc ratio of 0.7 or higher, a cup-disc ratio asymmetry exceeding 0.2 , or focal notching with compatible visual field loss upon automated static perimetry. All of the enrolled patients underwent UBM examinations before LPI, and the iris configuration based on four sites (horizontal and vertical) was an iris bow.

The exclusion criteria included eyes with posterior synechiae, extensive PAS $\left(>180^{\circ}\right)$, and gross iris atrophy; those with high myopia or hyperopia (spherical equivalent refractive error greater than +6 or -6 diopters), low-quality images due to clinically relevant opacities of the optic media, and unstable fixation; patients who received topical or systemic medication that could affect the iris or angle configuration; and eyes with previous histories of intraocular surgery.

\section{LPI}

All of the LPIs were performed by the same ophthalmologist (XJZ) and in accordance with our previous study [14]. Briefly, the LPI was performed using a VISULAS ${ }^{\otimes} 532 \mathrm{~s}$ diode laser (Carl Zeiss Meditec, Inc.). Thirty minutes prior to the procedure, pharmacological pupil constriction was induced with topical $2 \%$ pilocarpine and topical anesthesia was induced with a $0.5 \%$ proparacaine hydrochloride ophthalmic solution (Alcaine; Alcon Pharmaceuticals, Fort Worth, TX, USA) in the eye undergoing surgery. The selected treatment site was the superotemporal or superonasal region between the 10 and 11 o'clock or the 1 and 2 o'clock positions. The treatment was initiated with a pulse of 3-5 mJ, and the power was increased until patency was achieved, and the opening of the iris was $>0.1 \mathrm{~mm}$. The patency was determined by direct visualization of the posterior chamber.

\section{SS-OCT measurements}

The anterior chamber parameters were measured using a CASIA-OCT (SS-1000; Tomey Corporation, Nagoya, Japan) as described in previous studies [15]. The participants underwent OCT examinations while in a seated position in a dark room with all lights turned off $(0-11 \mathrm{x}$ ambient light). The images were obtained as a crosssectional full-circle scan (angles at $0-360^{\circ}$ ) with scans performed across the center of the pupil (Fig. 1a). Several scans were obtained for each subject, and the best image was selected for further analysis. Twelve images of the 12 clock positions ( 1 clock to 12 clock) were chosen for further analysis (as indicated by the yellow arrow shown in Fig. 1). The images were then processed using the angle assessment program in the machine. Images with artefacts caused by blinking or eye movements were not included in the data analysis. All of the OCT figures were assessed by two independent doctors (Dr. Fei Li and Dr. Kai Gao), and if the final iris configuration definition was inconsistent, a third doctor joined the discussion to make a final decision. All of the doctors were blinded to the diagnoses and whether the patient received LPI treatment when they were reading the OCT results.

\section{Iris configuration definition}

Iris bow was defined based on OCT results as described in our previous study, as follows [14]: determinations were 
Fig. 1 The measurement of the iris bow configuration based on figures obtained using OCT. The iris bow configuration was determined based on 12 clock positions. a, b Results obtained before LPI. c, d Results obtained after LPI. The red circle shows the site of LPI.
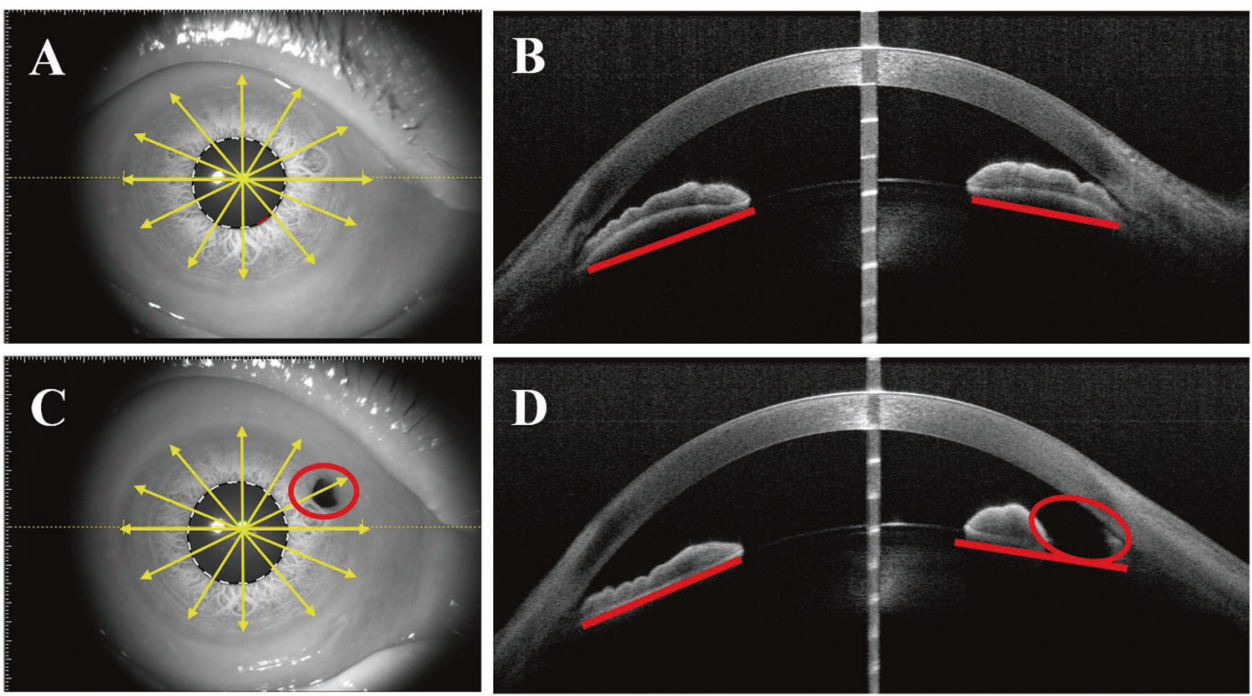

judged according to the curvature of the posterior surface of the iris and as the location of the posterior surface of the iris above a line drawn from the iris root to the iris margin of the pupil (Fig. 1b). The overall iris bow configuration of the eye was classified as follows:

1. When defined based on 12 clock positions, if $>6$ clock positions were iris bow, the whole eye was considered to be iris bow. If exactly 6 clock positions were iris bow (and the other 6 were not), the eye was described as having a coexisting configuration.

2. When defined based on 4 clock positions (horizontal and vertical sites, including the 3, 6, 9, and 12 o'clock positions), if $>2$ clock positions were iris bow, the eye was considered to be iris bow. If exactly 2 clock positions were iris bow (and the other two were not), the eye was described as having a coexisting configuration.

\section{Statistical analysis}

Data are presented as the mean \pm standard deviation for continuous variables and as the frequency (percentage) for categorical variables. Cronbach's alpha was used to measure internal consistency between measurements obtained at 12 sites and 4 sites, and the commonly accepted rule of internal consistency was: $<0.5$, unacceptable; $0.5-0.6$, poor; 0.6-0.7, questionable; 0.7-0.8, acceptable; 0.8-0.9, good; and $\geq 0.9$, excellent. Cronbach's alpha was also applied to compare changes in the iris configuration between before and after surgery. Pearson's correlation analysis was used to evaluate the relationships between changes in iris configuration and both age and pupil diameter. In addition, the rate of iris bow change from before to after surgery was compared using a chi-squared test. The $t$-test was used to compare the changes in iris bow at different LPI locations. All of the data were processed and analyzed using the Statistical Package for the Social Sciences (version 17.0; SPSS Inc., Chicago, IL, USA). For all of the tests, a twosided $p$ value $<0.05$ was considered statistically significant.

\section{Results}

\section{Demographic and clinical characteristics of the patients}

A total of 91 patients were enrolled in this study, and the subjects' demographic data are shown in Table 1. There were 61 PACS eyes and 30 PAC/PACG eyes. All 91 of the patients were followed up 1 week after LPI, and 87 patients (93.5\%) returned 2-4 months after LPI. We enrolled 47 patients who returned for re-examinations exactly 3 months after LPI for further analysis. The mean age of the enrolled patients was $60.7 \pm 8.72$ years old, and more female patients $(\sim 74.7 \%)$ than male patients were enrolled in this study, in accordance with the higher incidence of closed angle glaucoma in females. Approximately $16.5 \%$ of the patients underwent LPI in the superonasal region, and $83.5 \%$ of the patients underwent LPI in the superotemporal region. The mean IOP was $14.7 \pm 3.4 \mathrm{mmHg}$ before LPI, $14.9 \pm 3.2 \mathrm{mmHg}$ one week after LPI, and $16.1 \pm 3.7 \mathrm{mmHg}$ 3 months after LPI, and these differences were not significantly different (all $p>0.05$ ).

\section{Change in the iris bow after LPI}

All of the patients enrolled in this study showed anterior iris bowing at all 12 clock positions, but only one 
patient showed iris flattening at the 10 and 12 o'clock positions. One week after LPI, an average of $40.14 \%$ of the clock positions still showed anterior iris bowing, and $245.9 \%$ showed anterior iris bowing at 3 months after LPI. When only 4 clock positions (3, 6, 9, and 12 o'clock) were considered, $40.9 \%$ and $46.3 \%$ of the clock positions showed anterior iris bowing at 1 week and 3 months after LPI, respectively. The changes in each position based on 12 clock positions were consistent (good to excellent) at 1 week and 3 months after LPI, with both having a Cronbach's alpha value of $>0.8$ (Table 2)

\section{Consistency of defining the iris bow using different measurements}

At baseline, almost all 12 of the positions exhibited anterior iris bowing; therefore, there was excellent consistency when the iris bow was assessed based on either 12 clock positions or 4 clock positions. After LPI, when evaluating the iris bow based on 12 clock positions, $34.1 \%$ of the eyes exhibited iris bow, and 3.3\% of the eyes exhibited a coexisting configuration 1 week after LPI. The iris bow statuses of the eyes were similar at 3 months after LPI, but at that timepoint, there were more coexisting configuration eyes $(10.6 \%)$ and fewer noniris bow eyes $(55.3 \%)$ than were observed at 1 week. When evaluating iris bow based on 4 clock positions, $33.0 \%$ and $31.9 \%$ of the eyes exhibited iris bow at 1 week and 3 months, respectively, and these findings were not significantly different from results based on 12 clock positions (both $p>0.05$ ); however, there were more coexisting configurations when using 4 clock positions than when using 12 clock positions $(16.5 \%$ vs. $3.3 \%$ at 1 week and $25.5 \%$ vs. $10.6 \%$ at 3 months). The consistency of the assessment methods was poor after LPI (Cronbach's alpha $=0.6$ at 1 week and 0.53 at 3 months); however,

Table 1 Baseline characteristics

\begin{tabular}{ll}
\hline Characteristics & Mean $(\mathrm{SD}) / n(\%)$ \\
\hline Eyes, $n(\%)$ & 91 \\
Right eye & $51(56.0)$ \\
Lift eye & $40(44.0)$ \\
Age (yr), mean (SD) & $60.7(8.72)$ \\
Female, $n(\%)$ & $68(74.7)$ \\
IOP (mmHg) & $14.7(3.4)$ \\
PD (mm), mean (SD) & $4.07(1.16)$ \\
LPI site, $n(\%)$ & \\
Superonasal & $15(16.5)$ \\
Superotemporal & $76(83.5)$ \\
\hline
\end{tabular}

$P D$ pupil diameter at baseline, $I O P$ intraocular pressure, $L P I$ site the location of laser peripheral iridotomy, $S D$ standard deviation when the coexisting configurations were excluded, the consistency between the two assessment methods was good to excellent (Cronbach's alpha $=0.91$ at 1 week and 0.85 at 3 months) (Table 3).

\section{Factors influencing the iris bow changes}

In this study, the effects of the different LPI locations were evaluated. As shown in Table 4, the average change rate from iris bow to non-iris bow was $66.58 \%$ when the LPI location was at 1 or 2 o'clock and $60.23 \%$ when the LPI location was at 10 or 11 o'clock after 1 week, and these results were not significantly different $(p=0.509)$. Three months after LPI, the average change in iris bow remained similar at the two different LPI locations $(64.06 \%$ vs. $62.05 \%$, respectively, $p=0.707)$. As shown in Supplementary Fig. 1, relieving the anterior iris bowing after LPI was more common at the LPI location site; however, the changes located at the other clock positions were relatively consistent, and the anterior iris bowing relief rate ranged

Table 2 The change of Iris's bow before and after surgery

\begin{tabular}{|c|c|c|c|}
\hline & $\begin{array}{l}\text { Baseline } \\
(n=91, \%)\end{array}$ & $\begin{array}{l}1 \text { week } \\
\text { after LPI } \\
(n=91, \%)\end{array}$ & $\begin{array}{l}3 \text { months } \\
\text { after LPI } \\
(n=47, \%)\end{array}$ \\
\hline \multicolumn{4}{|l|}{ Among 12 sites } \\
\hline At $12: 00$ o'clock & $90(98.9)$ & $37(40.7)$ & $15(31.9)$ \\
\hline At 11:00 o'clock & $91(100)$ & $25(27.5)$ & $16(34.0)$ \\
\hline At 10:00 o'clock & $90(98.9)$ & $26(28.6)$ & $18(38.3)$ \\
\hline At 9:00 o'clock & $91(100)$ & $38(41.8)$ & $27(57.5)$ \\
\hline At 8:00 o'clock & $91(100)$ & $51(56.0)$ & $32(68.1)$ \\
\hline At 7:00 o'clock & $91(100)$ & $42(46.2)$ & $23(48.9)$ \\
\hline At 6:00 o'clock & $91(100)$ & $35(38.5)$ & $17(36.2)$ \\
\hline At 5:00 o'clock & $91(100)$ & $36(39.6)$ & $25(53.2)$ \\
\hline At 4:00 o'clock & $91(100)$ & $44(48.4)$ & $24(51.1)$ \\
\hline At 3:00 o'clock & $91(100)$ & $39(42.9)$ & $28(59.6)$ \\
\hline At 2:00 o'clock & $91(100)$ & $36(39.6)$ & $18(38.3)$ \\
\hline At 1:00 o'clock & $91(100)$ & $29(31.9)$ & $16(34.0)$ \\
\hline Average (\%) & 99.80 & 40.14 & 45.9 \\
\hline Cronbach's alpha & $\mathrm{n} / \mathrm{a}$ & 0.91 & 0.89 \\
\hline \multicolumn{4}{|l|}{ Among four sites } \\
\hline At 3:00 o'clock & $91(100)$ & $39(42.9)$ & $28(59.6)$ \\
\hline At 6:00 o'clock & $91(100)$ & $35(38.5)$ & $17(36.2)$ \\
\hline At 9:00 o'clock & $91(100)$ & $38(41.8)$ & $27(57.5)$ \\
\hline At $12: 00$ o'clock & $90(98.9)$ & $37(40.7)$ & $15(31.9)$ \\
\hline Average $(\%)$ & 99.70 & 40.90 & 46.3 \\
\hline Cronbach's alpha & $\mathrm{n} / \mathrm{a}$ & 0.81 & 0.67 \\
\hline
\end{tabular}

Data were presented as $n(\%), n$ stands for the number of iris bow configuration before and after surgery. n/a: almost all 12 of the positions exhibited anterior iris bowing, therefore, there was no Cronbach's alpha data at baseline 
Table 3 The distribution of Iris's configuration after surgery among different locations

\begin{tabular}{|c|c|c|c|c|c|c|}
\hline & \multicolumn{3}{|c|}{1 week after LPI } & \multicolumn{3}{|c|}{3 months after LPI } \\
\hline & Iris bow & Co-existing & Non-iris bow & Iris bow & Co-existing & Non-iris bow \\
\hline Among 12 sites & $31(34.1 \%)$ & $3(3.30 \%)$ & $57(62.6 \%)$ & $16(34.0 \%)$ & $5(10.6 \%)$ & $26(55.3 \%)$ \\
\hline Among 4 sites & $30(33.0 \%)$ & $15(16.5 \%)$ & $46(50.5 \%)$ & $15(31.9 \%)$ & $12(25.5 \%)$ & $20(42.6 \%)$ \\
\hline Cronbach's alpha ${ }^{a}$ & 0.6 & & & 0.53 & & \\
\hline Cronbach's alphab & 0.91 & & & 0.85 & & \\
\hline
\end{tabular}

Data were presented as $n(\%)$. Co-existing: among 12 sites, there are 6 sits were iris bombe and 6 sites were non iris bow; among 4 sites, there are 2 sites were iris bow and 2 sits were non iris bow

acronbach's alpha: Cronbach's alpha between the distribution of 4 and 12 directions including all the types ${ }^{\text {b}}$ Cronbach's alpha: Cronbach's alpha between the distribution of 4 and 12 directions excluding mixed types

\begin{tabular}{|c|c|c|c|c|c|c|c|c|c|c|c|c|}
\hline & \multicolumn{3}{|c|}{$\begin{array}{l}\text { 1:00 or } 2: 00 \text { o'clock } \\
(n=43)\end{array}$} & \multicolumn{3}{|c|}{$\begin{array}{l}\text { 10:00 or } 11: 00 \text { o'clock } \\
(n=48)\end{array}$} & \multicolumn{3}{|c|}{$\begin{array}{l}\text { 1:00 or } 2: 00 \text { o'clock } \\
(n=22)\end{array}$} & \multicolumn{3}{|c|}{$\begin{array}{l}\text { 10:00 or 11:00 o'clock } \\
(n=25)\end{array}$} \\
\hline & BA & $1 \mathrm{w}$ & $\Delta$ & BA & $1 \mathrm{w}$ & $\Delta$ & BA & $3 \mathrm{~m}$ & $\Delta$ & BA & $3 \mathrm{~m}$ & $\Delta$ \\
\hline $12: 00$ & 98.60 & 35.15 & 63.45 & 100.00 & 40.00 & 60.00 & 98.60 & 22.50 & 76.10 & 100.00 & 58.35 & 41.65 \\
\hline $11: 00$ & 100.00 & 26.60 & 73.40 & 100.00 & 20.70 & 79.30 & 100.00 & 30.00 & 70.00 & 100.00 & 22.50 & 77.50 \\
\hline $10: 00$ & 98.60 & 36.50 & 62.10 & 100.00 & 16.45 & 83.55 & 98.60 & 30.00 & 68.60 & 100.00 & 26.65 & 73.35 \\
\hline 9:00 & 100.00 & 30.75 & 69.25 & 100.00 & 42.15 & 57.85 & 100.00 & 40.00 & 60.00 & 100.00 & 39.15 & 60.85 \\
\hline 8:00 & 100.00 & 39.30 & 60.70 & 100.00 & 46.05 & 53.95 & 100.00 & 47.50 & 52.50 & 100.00 & 59.15 & 40.85 \\
\hline 7:00 & 100.00 & 28.00 & 72.00 & 100.00 & 53.55 & 46.45 & 100.00 & 40.00 & 60.00 & 100.00 & 30.85 & 69.15 \\
\hline 6:00 & 100.00 & 33.75 & 66.25 & 100.00 & 38.95 & 61.05 & 100.00 & 30.00 & 70.00 & 100.00 & 30.85 & 69.15 \\
\hline 5:00 & 100.00 & 36.50 & 63.50 & 100.00 & 37.15 & 62.85 & 100.00 & 45.00 & 55.00 & 100.00 & 49.15 & 50.85 \\
\hline 4:00 & 100.00 & 47.85 & 52.15 & 100.00 & 45.70 & 54.30 & 100.00 & 37.50 & 62.50 & 100.00 & 35.00 & 65.00 \\
\hline 3:00 & 100.00 & 29.35 & 70.65 & 100.00 & 44.30 & 55.70 & 100.00 & 40.00 & 60.00 & 100.00 & 39.15 & 60.85 \\
\hline 2:00 & 100.00 & 21.05 & 78.95 & 100.00 & 52.50 & 47.50 & 100.00 & 30.00 & 70.00 & 100.00 & 26.65 & 73.35 \\
\hline 1:00 & 100.00 & 16.85 & 83.15 & 100.00 & 43.95 & 56.05 & 100.00 & 17.50 & 82.50 & 100.00 & 16.65 & 83.35 \\
\hline Average & 99.75 & 33.16 & 66.58 & 100.00 & 39.77 & 60.23 & 99.75 & 35.68 & 64.06 & 100.00 & 37.95 & 62.05 \\
\hline$p$-value & $0.509^{*}$ & & & & & & $0.707^{\#}$ & & & & & \\
\hline
\end{tabular}

Data were presented as $\%$

$B A$ baseline, $1 w 1$ week after LPI, $3 m 3$ months after LPI, $\Delta$ mean change

* $p$ value: comparison of average change of iris bow between different LPI location at 1 week

${ }^{\#} p$ value: comparison of average change of iris bow between different LPI location at 3 months from $50 \%$ to $70 \%$. Age and pupil diameter at baseline had limited effects on iris configuration changes (both $p>0.05$ ).

\section{Discussion}

A few studies have been performed to explore the changes in the anterior segment parameters that occur after LPI in patients with angle closure eyes [10]. However, to date, all of those studies have focused on horizontal or vertical anterior segment parameters. In this study, we used anterior SS-OCT to fully explore the iris bow changes that occurred after LPI using 12 clock positions, and we found that iris bow changes were consistent after LPI at each clock position except the LPI site. There was excellent consistency when comparing iris bow evaluations based on measurements obtained at 12 clock positions and those obtained at 4 clock positions; however, there were more coexisting iris configurations when measurements were assessed based on 4 clock positions. After 3 months of follow-up, none of the patients whose iris bow was not relieved by LPI exhibited increasing IOP or suffered from an acute angle closure crisis.

AS-OCT has been demonstrated to be valuable for assessing iris structures, such as the iris curvature and concave iris configuration, that are involved in different eye diseases, including angle closure disease and pigment dispersion syndrome [16-18]. However, among these studies, only the horizontal or vertical meridian $(3,6,9$, and 12 o'clock positions) was selected to evaluate iris structures. 
Four sites cannot represent the whole structure of the iris, especially in angle closure eyes. In our study, we measured 12 clock positions in one eye, and we compared the iris bow configurations as defined based on these 12 clock positions versus only 4 clock positions. The methods showed that there was excellent consistency with regard for judging iris bow; however, there were more coexisting configurations when the eyes were assessed using only 4 clock positions than there were when 12 clock positions were used. Therefore, more attention should be paid to the final definition of iris configuration when it is assessed using only 4 clock positions with OCT or UBM.

In our study, $\sim 30 \%$ of the patients still exhibited iris bow after LPI, consistent with the results of other studies. Several researchers have found that the mean iris curvature value was reduced after LPI when AS-OCT was used to measure anterior chamber angle parameters. However, the iris curvature value remained positive after LPI, indicating that the iris bow remained after LPI $[10,19,20]$. One study evaluated the anterior angle using gonioscopy before and after LPI in 244 subjects with PACS or PAC/PACG. After LPI, $77 \%$ of the PACS eyes and $52.4 \%$ of the PAC/PACG eyes had two or more angle opening quadrants, further demonstrating that LPI only relieved part of the iris bow configuration [21]. Additionally, Lee et al. found that in $23.9 \%$ of narrow angle eyes assessed by gonioscopy and $34.8 \%$ of narrow angle eyes assessed by AS-OCT in the temporal quadrant retained persistent angle closure after LPI [9]. All of these studies (including our study) indicate that LPI partly relieves anterior iris bowing and that the relief of iris bow was the main factor that led to anterior chamber angle widening. A longer follow-up time is needed to determine what occurs in patients whose iris bow is not relieved by LPI. In our study, our follow-up duration was 3 months, and none of the patients exhibited an increasing IOP or suffered from an acute angle closure crisis.

We also compared the effects of LPI site on iris bow configuration changes. The LPI sites in all of the patients enrolled in this study were located superiorly (either superonasally or superotemporally). There was no significant difference between these two sites with regard for iris bow configuration changes. However, anterior iris bowing located around the LPI site was relieved more often than bowing located at the other clock positions. Therefore, when considering the effects of LPI on the relief of anterior iris bowing, both superonasal and superotemporal locations are suitable, and results depend on whether there is an iris cyst, a thin area in the iris, or iris blood, which requires less energy and reduces the chance of bleeding. Unfortunately, we did not have any patients whose LPI site was located in the inferior part of the iris. One study found that inferior LPI required fewer laser pulses and led to smaller IOP increases after 12 months of follow up [22]. However, Srinivasan et al. found no significant differences in the use of postoperative IOP-lowering drops or the amount of laser energy used for LPI sites located superiorly versus nasally/temporally [23]. However, neither study measured iris configuration changes, and further studies are therefore needed to explore the clock positions of iris configuration and anterior chamber parameters when different LPI locations are used.

This study did have some limitations. First, it included a relatively small sample size; in particular, the unequal distribution of subjects in the PACS and PAC/PACG groups was a limitation of this study and may have influenced the results of defining the iris bow configuration after LPI. However, at baseline, the iris bow configuration was consistent in each group. Second, we only enrolled angle closure eyes with anterior iris bowing, and this may have influenced the consistency of judgements between different methods. However, in this study, we also evaluated the iris structure after LPI procedures that relieved part of the anterior iris bowing, and this could have eliminated this bias. Third, we followed these patients for only 3 months after LPI, and none of the patients exhibited an increased IOP or acute angle closure crisis during this time. Therefore, we cannot make a definitive conclusion about what would happen to the $30 \%$ of the patients whose iris bow was not relieved by LPI. A longer follow-up period is needed to determine the prognosis of these patients. Finally, we only assessed the iris structure (anterior iris bowing) in this study, and we did not measure anterior chamber angle parameters, such as iris thickness and angle opening distance, and these parameters may be more directly relevant to the closure of the anterior chamber angle.

In conclusion, the results of this study show that there was excellent consistency when assessing iris bow using 12 clock positions and 4 clock positions; therefore, in a clinical setting, it is reasonable to define iris bow based on horizontal and vertical positions (that is, four clock sites) on figures obtained by UBM or OCT. However, there were more coexisting configurations when the 4 clock positions were used. Moreover, a single LPI relieved $\sim 2 / 3$ of all iris bow configurations and caused the unified relief of anterior iris bowing at all 12 clock positions except the iridotomy site.

\section{Summary}

\section{What was known before}

- Iris configuration can be measured by UBM or OCT, however, whether the iris bow defined from 4 clock positions (3, 6, 9, and 12 o'clock) stand for 12 clock positions and how the change of iris bow after LPI in in primary angle closure eyes remains unknown. 


\section{What this study adds}

- In this cohort observational study of 93 primary angle closure eyes, it was excellent consistency when measuring the iris bow in 4 and 12 clock positions.

- LPI caused a relative unified iris bow change and relieved about $2 / 3$ of the iris bow configurations.

Acknowledgements We thank Dr. XiaoJing Zhong, who helped perform the LPI procedure, and Dr. Guangxian Chen, who helped perform the OCT for all the enrolled patients.

Funding This study was supported by the National Natural Science Foundation of China $(81600728,81670847)$ and the Science and Technology Program of Guangzhou, China (201803010066).

\section{Compliance with ethical standards}

Conflict of interest The authors declare that they have no conflict of interest.

Publisher's note Springer Nature remains neutral with regard to jurisdictional claims in published maps and institutional affiliations.

\section{References}

1. Henzan IM, Tomidokoro A, Uejo C, Sakai H, Sawaguchi S, Iwase A, et al. Comparison of ultrasound biomicroscopic configurations among primary angle closure, its suspects, and nonoccludable angles: the Kumejima Study. Am J Ophthalmol. 2011;151:1065-.e1.

2. Radhakrishnan S, Goldsmith J, Huang D, Westphal V, Dueker DK, Rollins AM, et al. Comparison of optical coherence tomography and ultrasound biomicroscopy for detection of narrow anterior chamber angles. Arch Ophthalmol. 2005;123:1053-9.

3. Nongpiur ME, Sakata LM, Friedman DS, He M, Chan YH, Lavanya $\mathrm{R}$, et al. Novel association of smaller anterior chamber width with angle closure in Singaporeans. Ophthalmology. 2010;117:1967-73.

4. Aptel F, Denis P. Optical coherence tomography quantitative analysis of iris volume changes after pharmacologic mydriasis. Ophthalmology. 2010;117:3-10.

5. Saw SM, Gazzard G, Friedman DS. Interventions for angleclosure glaucoma: an evidence-based update. Ophthalmology. 2003;110:1869-78. quiz1878-1879, 1930

6. Ang LP, Aung T, Chew PT. Acute primary angle closure in an Asian population: long-term outcome of the fellow eye after prophylactic laser peripheral iridotomy. Ophthalmology. 2000;107:2092-6.

7. Lai J, Choy BN, Shum JW. Management of primary angle-closure glaucoma. Asia Pac J Ophthalmol (Philos). 2016;5:59-62.

8. Choi JS, Kim YY. Progression of peripheral anterior synechiae after laser iridotomy. Am J Ophthalmol. 2005;140:1125-7.

9. Lee KS, Sung KR, Kang SY, Cho JW, Kim DY, Kook MS. Residual anterior chamber angle closure in narrow-angle eyes following laser peripheral iridotomy: anterior segment optical coherence tomography quantitative study. Jpn J Ophthalmol. 2011;55:213-9.

10. How AC, Baskaran M, Kumar RS, He M, Foster PJ, Lavanya R, et al. Changes in anterior segment morphology after laser peripheral iridotomy: an anterior segment optical coherence tomography study. Ophthalmology. 2012;119:1383-7.

11. Zheng C, Guzman CP, Cheung CY, He Y, Friedman DS, Ong SH, et al. Analysis of anterior segment dynamics using anterior segment optical coherence tomography before and after laser peripheral iridotomy. JAMA Ophthalmol. 2013;131:44-49.

12. Memarzadeh F, Li Y, Chopra V, Varma R, Francis BA, Huang D. Anterior segment optical coherence tomography for imaging the anterior chamber after laser peripheral iridotomy. Am J Ophthalmol. 2007;143:877-9.

13. He M, Friedman DS, Ge J, Huang W, Jin C, Cai X, et al. Laser peripheral iridotomy in eyes with narrow drainage angles: ultrasound biomicroscopy outcomes. The Liwan Eye Study. Ophthalmology. 2007;114:1513-9.

14. Chen S, Lv J, Fan S, Zhang H, Xie L, Xu L, et al. Laser peripheral iridotomy versus laser peripheral iridotomy plus laser peripheral iridoplasty in the treatment of multi-mechanism angle closure: study protocol for a randomized controlled trial. Trials. 2017; 18:130

15. Gao K, Li F, Aung T, Zhang X. Diurnal variations in the morphology of Schlemm's canal and intraocular pressure in healthy Chinese: an SS-OCT study. Invest Ophthalmol Vis Sci. 2017;58:5777-82.

16. Schuster AK, Fischer JE, Vossmerbaeumer U. Curvature of iris profile in spectral domain optical coherence tomography and dependency to refraction, age and pupil size - the MIPH Eye \& Health Study. Acta Ophthalmol. 2017;95:175-81.

17. Liu L, Ong EL, Crowston J. The concave iris in pigment dispersion syndrome. Ophthalmology. 2011;118:66-70.

18. Nonaka A, Iwawaki T, Kikuchi M, Fujihara M, Nishida A, Kurimoto Y. Quantitative evaluation of iris convexity in primary angle closure. Am J Ophthalmol. 2007;143:695-7.

19. Moghimi S, Bijani F, Chen R, Yasseri M, He M, Lin SC, et al. Anterior segment dimensions following laser iridotomy in acute primary angle closure and fellow eyes. Am J Ophthalmol. 2018; 186:59-68.

20. Moghimi S, Chen R, Johari M, Bijani F, Mohammadi M, Khodabandeh A, et al. Changes in anterior segment morphology after laser peripheral iridotomy in acute primary angle closure. Am J Ophthalmol. 2016;166:133-40.

21. Zebardast N, Kavitha S, Krishnamurthy P, Friedman DS, Nongpiur ME, Aung T, et al. Changes in anterior segment morphology and predictors of angle widening after laser iridotomy in south Indian eyes. Ophthalmology. 2016;123:2519-26.

22. Ahmadi M, Naderi BZ, Naderi BA, Kianersi F. Efficacy of neodymium-doped yttrium aluminum garnet laser iridotomies in primary angle-closure diseases: superior peripheral iridotomy versus inferior peripheral iridotomy. Curr Med Res Opin. 2017;33:687-92.

23. Srinivasan K, Zebardast N, Krishnamurthy P, Abdul KM, Raman $\mathrm{GV}$, Rajendrababu S, et al. Comparison of new visual disturbances after superior versus nasal/temporal laser peripheral iridotomy: a prospective randomized trial. Ophthalmology. 2018;125:345-51. 